\title{
The application research of cloud computing on mobile communication
}

\author{
Zhiming Yang ${ }^{1, a}$, Fang Yin ${ }^{2, b}$ \\ ${ }^{1}$ College of Information Science, Yunnan University of Finance and Economics, Kunmiong, 650221, \\ China \\ ${ }^{2}$ College of Energy and Environment Science, Yunnan Normal University, Kunming, 650500, China \\ aemail:kmyzm@sina.com, bemail:yf6709@sina.com
}

Keywords: Cloud computing; Virtuality; Server; Internet; Mobile communication

\begin{abstract}
Cloud computing is a platform based on the Internet, which can provide application service of a new calculation model for customers, and socialize computing service. Cloud computing will have a positive impact on the development of mobile communication due to its safe and reliable data storage and powerful computing ability. This paper analyzes the connotation, characteristics, and mechanism of cloud computing, as well as the positive role of cloud computing during mobile communication development combined with mobile communication service.
\end{abstract}

\section{Definition of Cloud Computing}

Cloud computing is a kind of super calculation mode based on internet, which set calculation task on resources pool constituted by a large number of computers, in order to make all kinds of application system to get calculating power, storage space, and different software maintenance as needed. Cloud computing is essentially to access applications or services via Internet, provided by the third parties instead of running on its own server. Its target is to put every calculation on the network. The cloud is network, and the network is computer. Relying on its powerful computation ability, the cloud computer can effectively utilize hardware platform connected by network, to carry out a variety of applications which make tens of thousands of end users no worry about the use of computing technology and the way of access. The cloud calculation's novel is that it can provide almost unlimited cheap storage and computing power. As a family electricity supply mode, it transforms the early single generator family power supply mode to more generation power concentrated social mode. It means that the computing power also can act as a kind of commodity for circulation.

\section{Characteristics of Cloud Computing}

The cloud computing has the following features, which can be used as a way of defining the concept. (1) The cloud computing can't be operated on single computer. It must be connected to Internet and added to suitable medium scale. (2) The cloud computing can be extended, which means it can complete quickly extension of hardware and server scalability according to calculation peak demand. (3) Cloud computing service may be cheap. Any technology popularity should be considered its cost investment. (4) Cloud computing should has a strong feasibility and virtualization. We choose cloud computing attribute to its convenient. People can enjoy the powerful and magical cloud computing by convenient use and common modify at anytime and anywhere.

\section{A kind of New Commercialize Model}

Cloud computing provides software and service via Internet, and implements by web browser. Users can join cloud computing and get access of browser at random with no need to install any server or client software at anytime, anywhere, and any device (with premise of connected to the Internet). The typical cloud computing service has three patterns, "software as a service (SaaS) SoftwareasaService,", platform as a service (PaaS) PlatformasaService, "and" infrastructure as a 
service "(IaaS) InfrastructureasaService. SaaS refers to use internet software via a standard web browser. From the user's point of view, this means that they don't need to cost on the server or software license authorization. From the supplier's point of view, this means a relative low software maintenance cost with conventional software service model. SaaS supplies usually collect fees by renting customer the software module, so users can order software application services according to their demand, and SaaS commerce chamber will be responsible for the deployment, upgrade, and maintenance of the system.

\section{Application of Cloud Computing in Mobile Communication}

(1) Enhancement of IT and Telecommunications Technology

Mobile Internet is a kind of core technology based on broadband IP technology, which can provide open basic telecommunication network at the same time, such as voice, data, multimedia, etc.. From the view point of user, the mobile Internet refers to use mobile terminals, such as mobile phones and laptops, to get access to the Internet by HTTP protocol and wireless mobile network. Under the background of cloud computing, IT and telecommunication technology will be accelerated the process of integration. The mobile Internet is an important field. Mobile devices will become expanding cloud service of remote controller, as well as rapid growth of different types in quantity and quality based on cloud connection.

(2) Reduction of Requirement of Mobile Terminal

Cloud computing will pour a powerful impetus into the development of mobile Internet. Mobile end devices are generally less storage capacity and weak computing power. Cloud computing transfer the "calculation" and mass data storage from the terminal to the server, which reduces the processing requirements on mobile end devices. In such way, mobile terminal will undertake user interaction function, while complex calculation is taken up by cloud side, also named server side. Mobile terminal can respond to user terminal operations without powerful operation ability, and ensure the user good experience, so as to realize the SaaS supported by cloud. Cloud computing brings down the demand for network. For example, when users need to look over a file, it is needed to send user the part of the content they wanted, without need to transfer the entire file to the user. Mobile terminal don't sense applied specific implementation, the expansion application come true the implementation and deployment in powerful server-side, and the interaction with user in mobile terminal in the same way, for example via a browser, so the expansion application becomes much easier. In a word, mobile communication will gradually step into the era of "big data", the custom-made data mining and user personalized service will become a growing trend. The theory and technology of cloud computing will undoubtedly has a positive role in mobile communication.

\section{Conclusion}

Cloud computing is a platform based on the Internet, which can provide application service of a new calculation model for customers, and socialize computing service. Cloud computing will have a positive impact on the development of mobile communication due to its safe and reliable data storage and powerful computing ability.

\section{Acknowledgement}

This paper was sponsored by the Nature Science Foundation of China (Project No. 51366015).

\section{References}

[1] Miller M. Cloud Computing: Web-Based Applications That Change the Way You Work and Collaborate Online. August 2008 
[2] Galen G. What Cloud Computing Really Means. April 7th 2008.

[3] Buyya R, Yeo C S, Venugopal S. Market-oriented Cloud Computing: Vision, Hype, and Reality for DeliveringIT Services as Computing and Communications, 2008.

[4] Grossman R L, Gu Y H, Sabala M, et al. Compute and Storage Clouds Using Wide Area High Performance Networks. CoRR, August 12th 2008.

[5] Kim, D.J. and Y. Hwang. A Study of Mobile Internet User's Service Quality Perception from a User's Utilization and Hedonic Value Tendency Perspectives. Information Systems Frontiers 14(2): 409-421.

[6] Gartner: Seven cloud-computing security risks, http://www.infoworld.com/ article/2652198/ security/gartner-seven-cloud-computing-security-risks.html. 